\section{Anemia no binômio mãe-filho no Estado de Pernambuco, Brasil}

\author{
Mother-child anemia in the State of Pernambuco, \\ Brazil
}

\begin{abstract}
1 Instituto Fernandes
Figueira, Fundação Oswaldo Cruz, Rio de Janeiro, Brasil.

2 Centro de Pesquisas Aggeu

Magalhães, Fundação

Oswaldo Cruz, Recife, Brasil.

3 Departamento de Nutrição,

Universidade Federal de

Pernambuco, Recife, Brasil.

4 Instituto de Medicina

Integral Prof. Fernando

Figueira, Recife, Brasil.

Correspondência

T. C. Miglioli

Instituto Fernandes Figueira,

Fundação Oswaldo Cruz.

Rua Leite Leal 135, bloco I,

apto. 405, Rio de Janeiro, $R J$

22240-100, Brasil.

tcmiglioli@terra.com.br
\end{abstract}

\begin{abstract}
Prevalence of anemia and associated factors were analyzed in mothers $(n=1,022)$ and their children under 5 years of age $(n=1,242)$ in Pernambuco State, Brazil, 2006. This was a cross-sectional population-based study with a probabilistic sample in an urban and rural area. Anemia was diagnosed by hemoglobin level (children $<11.0 \mathrm{~g}$ ) $d L$, women $<12.0 \mathrm{~g} / \mathrm{dL}$ ) using HemoCue. Univariate and multivariate analyses used Poisson regression with robust adjustment of standard error, adopting a hierarchical model for determination in children with anemia as the outcome. This same procedure was not applied to mothers because of the small number of associated factors in the univariate analyses. Anemia prevalence was $16.4 \%$ in mothers and $34.4 \%$ in children. Anemic as compared to non-anemic mothers showed a prevalence ratio of 1.44 (95\% CI: 1.211.72) for anemia in their children, maintaining similar values in the adjusted model $(P R=1.39$ : 95\%CI: 1.16-1.66). Anemia prevalence in children was double that of mothers, with the final model showing only one common factor: per capita family income.
\end{abstract}

Anemia; Mothers; Prevalence
Teresa Cristina Miglioli 1

Ana Maria de Brito 2

Pedro Israel Cabral de Lira ${ }^{3}$

José Natal Figueroa 4

Malaquias Batista Filho 4

\section{Introdução}

A anemia se distribui, com características endêmicas em todos os grupos socioeconômicos e segmentos biológicos, constituindo a mais disseminada das carências nutricionais, embora predomine nos contingentes mais pobres da população 1 .

Segundo informes de comitês de especialistas da Organização Mundial da Saúde (OMS), a deficiência de ferro seria o principal fator etiológico das anemias carenciais do mundo, no entanto, estudos mais atuais redimensionaram esta participação para cerca de 50\%, representando, portanto, uma mudança na interpretação do problema $1,2,3,4$

Crianças e mulheres em idade reprodutiva representam os grupos biológicos mais vulneráveis à ocorrência da anemia, mas, ainda assim, são raros os estudos abordando o problema no conjunto mães/filhos biológicos, bem como os fatores de risco, tanto em nível individual como familiar. É um dos problemas de saúde de caracterização mais antiga 5 que afeta cerca da metade de toda a população humana 1 , sendo a deficiência nutricional de maior prevalência da atualidade. Apesar disso, somente em 1990, com a Reunião de Cúpula das Nações Unidas, formalizou-se o primeiro compromisso internacional para a definição de um programa mínimo para seu controle 6 . Depois dessa reunião, ampliou-se 
consideravelmente o interesse da área de pesquisa, dos formuladores de políticas e gestores de programas e de projetos experimentais de intervenção sobre o problema, sem, no entanto, alcançar os avanços programados.

Em 1999, o governo, a sociedade civil, a comunidade científica e as indústrias brasileiras de massas alimentares firmaram o Compromisso Social para a Redução da Anemia por Carência de Ferro no Brasil 7. Como parte do compromisso assumido, foi formalizada, no ano de 2002, a Resolução $n^{o} .344^{8}$ da Agência Nacional de Vigilância Sanitária, tornando obrigatória a adição de ferro e ácido fólico nas farinhas de milho e trigo. Essa medida só entrou em vigor em junho de 2004, em função do prazo de dois anos estipulado para as empresas se adequarem às normas então estabelecidas.

O enfoque na educação alimentar para a redução da anemia foi outra estratégia adotada nesse compromisso, reforçando a necessidade da orientação alimentar e nutricional sob a ótica da Promoção da Alimentação Saudável 7. Em 2005, o Ministério da Saúde instituiu o Programa Nacional de Suplementação de Ferro com o objetivo de promover a suplementação universal de crianças de 6 a 18 meses, gestantes a partir da 20a semana e mulheres no pós-parto ${ }^{9}$.

Existem poucos estudos abordando, a partir de um enfoque familiar, as características de distribuição do estado nutricional e seus fatores de risco, referenciando os grupos biológicos mais vulneráveis aos problemas nutricionais, ou seja, crianças menores de cinco anos e mulheres no período reprodutivo. No caso do Brasil, citam-se os trabalhos de Engstrom \& Anjos 10,11 e Silva et al. 12 , sendo que apenas este último aborda o problema da anemia no binômio mãe-filho.

Assim, a abordagem do agregado familiar e, particularmente, do pareamento mãe-filho biológico, representa uma oportunidade muito peculiar de estudo, seja pela rapidez das mudanças produzidas no perfil nutricional, seja pelo interesse em compreender e atuar nas políticas públicas a partir de um enfoque familiar. Seriam os casos do Programa Saúde da Família (PSF) e da segurança alimentar do Programa Bolsa Família (PBF), que praticamente atendem a metade da população brasileira 13,14.

Nesse contexto, o estado de saúde do núcleo familiar mais exposto aos desvios nutricionais, crianças e mães, pode oferecer subsídios importantes sobre questões que interessam diretamente às políticas públicas dirigidas para a segurança alimentar e nutricional.

Objetivou-se investigar a prevalência de anemia em mães e filhos, no Estado de Pernambuco, e sua associação com fatores biológicos, espa- ciais, socioeconômicos e condições de acesso aos serviços.

\section{Metodologia}

Utilizou-se banco de dados da pesquisa Situação Alimentar Nutricional e de Saúde no Estado de Pernambuco: Contexto Socioeconômico e de Serviços - III Pesquisa Estadual de Saúde e Nutrição (III PESN/PE-2006), trata-se de um inquérito de corte transversal, de base populacional, com amostra probabilística representativa do meio urbano e rural do Estado de Pernambuco.

Tomou-se a criança como unidade amostral e para a definição da amostra foram considerados como unidade de observação, domicílios com menores de cinco anos de idade. Para o dimensionamento da amostra, foram utilizadas as prevalências de desnutrição estimadas há dez anos no estado, obtidas na II Pesquisa Estadual de Saúde e Nutrição 17, representadas por valores de $3,2 \%$ para o setor urbano, e $6,2 \%$, para o rural, usando o indicador peso/idade. A partir desse critério básico foram estimadas sub-amostras para representar estratos geográficos e grupos populacionais específicos.

O sorteio da unidade amostral (crianças menores de cinco anos) em cada um dos estratos se processou em três estágios: município, setor censitário e domicílio. Por meio de sorteio aleatório condicionado à probabilidade proporcional das respectivas populações, foram selecionados 18 municípios dos 185 existentes. Numa segunda etapa, foram sorteados, de forma aleatória sistemática, 20 setores censitários da área urbana, sendo 10 da Região Metropolitana do Recife e 10 de 13 municípios do interior do estado. Da área rural, foram sorteados 19 setores censitários entre os 13 municípios pesquisados. No total foram incluídos 39 setores censitários. Deve-se considerar que a relação entre os universos populacionais da Região Metropolitana do Recife $(49,4 \%)$ e do interior urbano $(50,6 \%)$ são praticamente iguais, dispensando, assim, a necessidade de ajustamento.

Em cada setor censitário sorteado foi tomado, como marco inicial para localização e identificação das crianças, o ponto extremo da face da quadra voltada para o nascente (critério utilizado pelo Instituto Brasileiro de Geografia e Estatística - IBGE) e, a partir desse ponto, no sentido horário, foram localizadas as unidades domiciliares onde residiam menores de cinco anos, estabelecendo-se, para cada setor, uma quota amostral de $45 \pm 5$ crianças.

A coleta dos dados foi realizada no período de 10 de maio a 25 de outubro de 2006, por 
uma equipe de campo familiarizada com inquéritos, utilizando questionários envolvendo informações em grande parte já estudadas em pesquisas anteriores no Estado de Pernambuco, observando-se critérios de validação de questionários testados na fase de capacitação do pessoal e experimentação da logística, consistência e integralidade dos instrumentos de coleta, mediante exercício piloto. Além disso, os questionários eram analisados pelos supervisores de campo e em seguida pelos coordenadores.

Do total de 1.650 crianças menores de cinco anos e 1.909 mulheres em idade fértil, analisadas na III PESN/PE-2006, para atender aos objetivos deste estudo, foram selecionadas as crianças de ambos os sexos, entre 6 e 59 meses de idade, que tiveram a dosagem de hemoglobina realizada, bem como suas mães biológicas, compondo assim o pareamento amostral mãe-filho.

Foram excluídas as crianças que estavam sob a responsabilidade de avós, tias ou o pai, e aquelas que tiveram processo de adoção, por não atenderem ao vínculo biológico de maternidade, adotado restritivamente como condição de interesse específico do estudo. Também foram excluídas as crianças cujas mães se encontravam grávidas ou que não forneceram informação a esse respeito. Em relação à gravidez, ressalta-se a particularidade de que a gestação aumenta entre $30 \%$ a $40 \%$ a ocorrência de anemia e, ademais, implica em fatores adicionais de risco, estabelecendo um possível viés para a representatividade da amostra e análise de seus resultados. Em função dessas considerações e critérios, foram estudadas 1.022 mães e 1.242 crianças menores de cinco anos.

A dosagem de hemoglobina foi realizada em fotômetro portátil HemoCue (Hemocue, Angelholm, Suécia). Para discriminação da anemia, como variável dependente, foram adotados os pontos de corte recomendados pela OMS 2: nível de hemoglobina abaixo de $12 \mathrm{~g} / \mathrm{dL}$ para as mães e abaixo de $11 \mathrm{~g} / \mathrm{dL}$ para menores de cinco anos. Como variáveis independentes foram consideradas variáveis biológicas, geográficas, socioeconômicas, acesso aos serviços de saneamento, de saúde e programas sociais, organizadas em blocos hierárquicos segundo a lógica de um modelo explicativo concebido para reunir e integrar as informações consideradas pertinentes, disponibilizadas no banco de dados. Essas variáveis estão nomeadas, agrupadas e ordenadas no modelo conceitual apresentado no ideograma da Figura 1.

A fim de se estabelecer as características da amostra, foram realizadas análises descritivas dos dados por meio de frequências absolutas e relativas.
A associação dos possíveis fatores preditivos da anemia foi inicialmente avaliada por análises univariadas, para mães e crianças, sendo selecionadas para o modelo multivariado, as variáveis que apresentaram nesta análise um valor de $\mathrm{p}<0,20$. As análises foram realizadas por meio de regressão de Poisson com ajuste robusto do erropadrão 18 e os resultados expressos por razão de prevalência (RP) e intervalo de 95\% de confiança (IC95\%). As associações foram avaliadas pela estatística teste de Wald.

As análises multivariadas foram realizadas distintamente para cada um dos grupos: mães e filhos.

Para as crianças, adotou-se um modelo teórico de determinação do desfecho, de acordo com literatura pesquisada 19,20, considerando a relação hierárquica entre as variáveis independentes. Assim, o modelo explicativo foi ordenado em três blocos, o primeiro formado pelas variáveis socioeconômicas e ambientais; o segundo, pelas variáveis relacionadas às mães e ao acesso aos serviços de saúde e programas sociais; no terceiro bloco figuraram as variáveis ligadas às crianças. Os fatores de risco foram avaliados após o ajuste para fatores de confusão de cada bloco e para aqueles hierarquicamente superiores. $\mathrm{Na}$ primeira etapa da análise foram introduzidas as variáveis do bloco I, sendo que as variáveis consideradas significantes neste bloco foram conservadas no modelo e entraram no ajuste do segundo bloco. As variáveis biológicas foram incluídas na análise dos dados na última etapa, por serem proximais em relação à determinação dos níveis de hemoglobina. As variáveis foram mantidas no modelo final ao apresentarem um nível de significância de 0,05.

Para as mães, pelo pequeno número de fatores associados à anemia na análise univariada, não se aplicou o procedimento estatístico hierárquico. A estratégia adotada para a seleção das variáveis foi do tipo backward.

Para a análise estatística foram utilizados os programas SPSS 8.0 (SPSS Inc., Chicago, Estados Unidos) e Stata 9.2 (Stata Corp., College Station, Estados Unidos). O projeto foi aprovado pelo Comitê de Ética em Pesquisa do Centro de Pesquisa Aggeu Magalhães (CPqAM/Fiocruz, sob o no. 116/2008).

\section{Resultados}

Foram estudadas 2.264 pessoas, incluindo mães e seus filhos menores de cinco anos, sendo 1.022 mulheres e 1.242 crianças, residentes em 1.013 domicílios, em nove dos quais mais de uma família dividia a mesma moradia. 
Figura 1

Modelo hierárquico do processo de determinação da anemia em crianças menores de cinco anos.

Bloco I

Fatores socioeconômicos

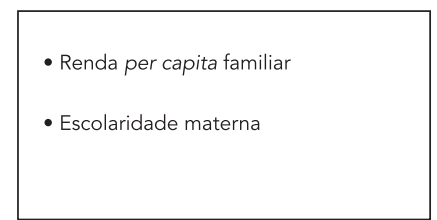

Fatores ligados ao domicílio

- Estrato geográfico

- Número de moradores por cômodo

- Caracterização do domicílio (regime de ocupação,

parede, piso)

- Acesso a serviços de saneamento (abastecimento

de água, esgotamento sanitário, destino do lixo)

\section{Bloco II}

Fatores maternos

Acesso a serviços de saúdee programas sociais

$$
\begin{aligned}
& \text { - Idade } \\
& \text { - Anemia } \\
& \text { - Número de consultas no pré-natal }
\end{aligned}
$$

- Visita do agente de saúde

- Cadastro no Programa Saúde Família

- Inscrição no Programa Bolsa Família

Bloco II

Fatores ligados à criança

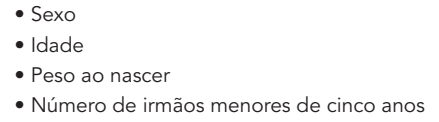

- Sexo

- Idade

- Peso ao nascer

- Número de irmãos menores de cinco anos

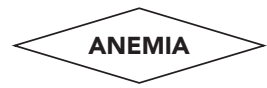

Do total de mães elegíveis para este estudo, 44 casos $(4,3 \%)$ não realizaram os exames laboratoriais, por recusa ou por não estar presente no momento da coleta de sangue. Assim, a dosagem de hemoglobina foi realizada em 978 mães, nas quais a prevalência de anemia foi de $16,4 \%$, com uma média de hemoglobina de 13,1g/dL (desviopadrão - DP = 1,3). Nas crianças a prevalência de anemia foi de $34,4 \%$, sendo a média de hemoglobina de 11,4g/dL (DP = 1,3) (Tabela 1).

Os resultados das análises univariadas entre as características relacionadas às condições biológicas, sócio-demográficas e ambientais e a ocorrência de anemia nas mães, encontram-se na Tabela 2, verificando-se que das 13 variáveis analisadas, apenas a inscrição no Programa Bolsa Família atuou com um valor limítrofe $(p=0,05)$ na ocorrência de anemia; ao lado de um valor marginal de $\mathrm{p}=0,06$ referente ao cadastro no PSF, e de $\mathrm{p}=0,07$ para a variável número de consultas pré-natais. Outras duas variáveis (renda familiar per capita, tipo de piso da moradia) com valores de significância abaixo de 20\%, também, foram selecionadas para a construção de modelos analíticos multivariados.

Em relação à escolaridade, a prevalência de anemia foi comparativamente mais elevada nas mulheres que tinham de 5 a 8 anos de estudo. As mães com renda familiar per capita abaixo de 0,25 salários mínimos apresentaram mais prevalência de anemia do que as mães do grupo de renda mais elevada, sem, no entanto essa diferença ser estatisticamente significante.

A ocorrência de anemia não apresentou associação estaticamente significativa entre mães do meio rural e urbano, número de pessoas por cômodos da habitação, tipo de parede ou de piso da moradia, acesso ao abastecimento de água, e, por fim, esgotamento sanitário. Apenas o tipo de piso (valor de $\mathrm{p}=0,19$ ), foi incluído na análise multivariada.

As mães que fizeram seis ou mais consultas pré-natais tiveram $15,5 \%$ de ocorrência de anemia, enquanto o grupo com três ou menos con- 
Tabela 1

Prevalência de anemia e médias de hemoglobina (Hb) em mães e seus filhos menores de cinco anos. Pernambuco, Brasil, 2006.

\begin{tabular}{lccc}
\hline Variáveis & $\mathbf{n}$ & $\begin{array}{c}\text { Prevalência } \\
\%(\text { (IC95\%) }\end{array}$ & $\begin{array}{c}\text { Hemoglobina (g/dL) } \\
\text { Média (DP) }\end{array}$ \\
\hline Condição das mães & & & \\
$\quad$ Anêmicas (Hb $<12 \mathrm{~g} / \mathrm{dL})$ & 160 & $16,4(14,0-18,7)$ & \\
$\quad$ Não anêmicas (Hb $\geq 12 \mathrm{~g} / \mathrm{dL})$ & 818 & $83,6(81,3-86,0)$ & $13,1(1,3)$ \\
$\quad$ Total & 978 & & \\
Condição dos filhos & & $34,4(31,7-37,0)$ & \\
Anêmicos (Hb $\geq 11 \mathrm{~g} / \mathrm{dL})$ & 427 & $65,6(63,0-68,3)$ & $11,4(1,3)$ \\
Não anêmicos $(\mathrm{Hb}<11 \mathrm{~g} / \mathrm{dL})$ & 815 & & \\
Total & 1242 & & \\
\hline
\end{tabular}

Tabela 2

Distribuição da anemia em mães de crianças menores de cinco anos segundo características biológicas, sócio-demográficas e ambientais. Pernambuco, Brasil, 2006.

\begin{tabular}{|c|c|c|c|c|}
\hline Variáveis analisadas & Amostra $(n=978)$ * & $\begin{array}{c}\text { Anemia } \\
n\end{array}$ & $\begin{array}{c}\text { RP (IC95\%) } \\
\%\end{array}$ & Valor de $p$ \\
\hline Idade (anos) & & & & 0,42 ** \\
\hline$<20$ & 110 & 20,9 & $1,33(0,86-2,05)$ & \\
\hline $20 \vdash 30$ & 512 & 15,4 & $0,95(0,70-1,30)$ & \\
\hline$\geq 30$ & 356 & 16,3 & 1,00 & \\
\hline Número de filhos menores de cinco anos & & & & 0,33 ** \\
\hline 1 & 778 & 17,1 & 1,00 & \\
\hline 2 & 184 & 13,0 & $0,76(0,51-1,14)$ & \\
\hline$\geq 3$ & 16 & 18,8 & $1,10(0,39-3,08)$ & \\
\hline Escolaridade (anos) & & & & 0,83 ** \\
\hline 0 & 60 & 15,0 & $1,06(0,54-2,08)$ & \\
\hline $1-4$ & 342 & 14,6 & $1,03(0,69-1,53)$ & \\
\hline $5-8$ & 319 & 20,4 & $1,44(0,99-2,09)$ & \\
\hline$\geq 9$ & 254 & 14,2 & 1,00 & \\
\hline 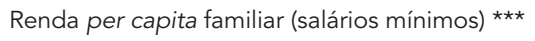 & & & & $0,18^{* \star}$ \\
\hline$<0,25$ & 484 & 17,6 & $2,28(0,76-6,89)$ & \\
\hline $0,25 \vdash 0,5$ & 307 & 15,3 & $1,99(0,65-6,09)$ & \\
\hline $0,5 \vdash 1,0$ & 129 & 16,3 & $2,12(0,67-6,72)$ & \\
\hline$\geq 1$ & 39 & 7,7 & 1,00 & \\
\hline Estrato geográfico & & & & 0,33 \\
\hline Urbano & 497 & 17,5 & 1,00 & \\
\hline Rural & 481 & 15,2 & $0,87(0,65-1,15)$ & \\
\hline Número de pessoas por cômodo & & & & 0,60 \\
\hline$<1$ & 428 & 14,7 & 1,00 & \\
\hline $1+2$ & 447 & 17,9 & $1,22(0,90-1,65)$ & \\
\hline $2+3$ & 72 & 20,8 & $1,42(0,85-2,35)$ & \\
\hline$\geq 3$ & 31 & 6,5 & $0,44(0,11-1,71)$ & \\
\hline Tipo de parede & & & & 0,57 \\
\hline Alvenaria/Tijolo & 914 & 16,2 & 1,00 & \\
\hline Outro & 64 & 18,8 & $1,16(0,68-1,97)$ & \\
\hline
\end{tabular}

(continua) 
Tabela 2 (continuação)

\begin{tabular}{|c|c|c|c|c|}
\hline Variáveis analisadas & Amostra $(n=978)$ * & $\begin{array}{c}\text { Anemia } \\
n\end{array}$ & $\begin{array}{c}\text { RP (IC95\%) } \\
\%\end{array}$ & Valor de $p$ \\
\hline Tipo de piso & & & & 0,19 \\
\hline Cerâmica/Lajota & 197 & 15,2 & 1,00 & \\
\hline Cimento & 729 & 15,9 & $1,04(0,72-1,51)$ & \\
\hline Outro & 52 & 26,9 & $1,77(1,01-3,08)$ & \\
\hline Abastecimento de água & & & & 0,34 \\
\hline Rede geral & 541 & 17,4 & 1,00 & \\
\hline Outro & 437 & 15,1 & $0,87(0,65-1,16)$ & \\
\hline Esgoto sanitário & & & & 0,63 \\
\hline Rede geral & 323 & 15,2 & 1,00 & \\
\hline Fossa com tampa & 327 & 17,4 & $1,15(0,81-1,63)$ & \\
\hline Outro & 326 & 16,6 & $1,09(0,77-1,56)$ & \\
\hline Número de consultas pré-natais & & & & 0,07 \\
\hline$\leq 3$ & 100 & 22,0 & $1,42(0,94-2,13)$ & \\
\hline $4-5$ & 193 & 18,7 & $1,20(0,85-1,70)$ & \\
\hline$\geq 6$ & 650 & 15,5 & 1,00 & \\
\hline Cadastro no Programa Saúde da Família & & & & 0,06 \\
\hline Sim & 664 & 16,4 & 1,00 & \\
\hline Não & 40 & 27,5 & $1,68(0,98-2,85)$ & \\
\hline Inscrição no Programa Bolsa Família & & & & 0,05 \\
\hline $\operatorname{Sim}$ & 703 & 14,9 & 1,00 & \\
\hline Não & 273 & 20,1 & $1,35(1,00-1,81)$ & \\
\hline
\end{tabular}

* Os totais parciais de cada grupo podem diferir do total da amostra em função de informações ignoradas pelas entrevistadas. No caso específico de cadastro no Programa Saúde da Família, 27,9\% da amostra residiam em áreas não atendidas pelo programa;

** Teste de tendência;

*** Salário mínimo na época: $\mathrm{R} \$ 350,00$.

sultas durante a gravidez teve uma prevalência de $22 \%$ de anemia ( $\mathrm{p}=0,07$ ) (Tabela 2 ).

Os resultados da análise de regressão multivariada realizada para o grupo de mães das crianças menores de cinco anos, apresentados na Tabela 3. Das cinco variáveis analisadas, apenas a renda per capita familiar e a inscrição no PBF se mantiveram no modelo explicativo final. A variável cadastro no PSF teve um efeito estatisticamente limítrofe com um valor de $\mathrm{p}=0,06$.

Em relação à distribuição da anemia nos menores de cinco anos, na análise univariada para os três blocos do modelo hierárquico, totalizando 16 variáveis, apenas as duas variáveis do bloco II (cadastro no PSF, inscrição no PBF) e duas do bloco III (sexo e peso ao nascer) apresentaram valores de $\mathrm{p}>0,20$, não sendo, assim, selecionadas para a construção de modelos analíticos multivariados. Verifica-se que a renda familiar per capita e a escolaridade das mães estavam associadas à prevalência de anemia dos filhos. Não houve diferença significativa nas taxas de anemia entre crianças das áreas urbana e rural.

Com exceção do abastecimento de água, todas as outras características físicas ou funcionais das moradias estiveram estatisticamente relacionadas com a ocorrência da anemia.

No grupo de "fatores maternos", todas as variáveis analisadas estavam associadas ao risco de anemia nos filhos. As maiores ocorrências de crianças anêmicas foram encontradas entre os filhos deadolescentes, mãesanêmicasequerealizaram menos de três consultas durante o pré-natal.

A condição de "cadastrado no Programa Saúde da Família” não apresentou associação com a ocorrência de anemia. O mesmo comportamento estatístico foi observado em relação à condição de estar ou não assistido pelo PBF.

Em relação ao sexo e ao peso ao nascer, não foram observadas diferenças estatísticas no que se refere à ocorrência de anemia entre as crianças.

Em referência à faixa etária, os resultados demonstraram tendência de declínio da prevalência de anemia com o aumento da idade das crianças. Assim, na faixa de 6 a 11 meses, o percentual de ocorrência de anemia foi de $63,4 \%$, enquanto nas crianças maiores (38 a 59 meses) a prevalência baixou para $12 \%$ (Tabela 4 ).

A Tabela 5 apresenta as RP bruta e ajustada entre a anemia e os diversos grupos de fatores 
Distribuição percentual da anemia e da razão de prevalência (RP) bruta e ajustada para as mães de crianças menores de cinco anos. Pernambuco, Brasil, 2006.

\begin{tabular}{|c|c|c|c|c|c|}
\hline Variáveis & $\begin{array}{c}\text { Anemia } \\
\%\end{array}$ & $\mathrm{RP}_{\text {bruta }}(\mathrm{IC} 95 \%)$ & Valor de $\mathrm{p}$ & $\mathrm{RP}_{\text {ajustada }}(\mathrm{IC} 95 \%)$ & Valor de $p$ \\
\hline Renda per capita familiar (salários mínimos) * & & & 0,18 ** & & 0,04 ** \\
\hline$<0,25$ & 17,6 & $2,28(0,76-6,89)$ & & $4,86(0,65-36,51)$ & \\
\hline $0,25 \vdash 0,5$ & 15,3 & $1,99(0,65-6,09)$ & & $3,55(0,47-26,63)$ & \\
\hline $0,5 \vdash 1,0$ & 16,3 & $2,12(0,67-6,72)$ & & $3,72(0,49-28,10)$ & \\
\hline$\geq 1$ & 7,7 & 1,00 & & 1,00 & \\
\hline Tipo de piso & & & 0,19 ** & & $0,47 \star *$ \\
\hline Cerâmica/Lajota & 15,2 & 1,00 & & 1,00 & \\
\hline Cimento & 15,9 & $1,04(0,72-1,51)$ & & $0,93(0,57-1,53)$ & \\
\hline Outro & 26,9 & $1,77(1,01-3,08)$ & & $1,71(0,81-3,63)$ & \\
\hline Número de consultas no pré-natal & & & 0,07 ** & & 0,16 ** \\
\hline$\leq 3$ & 22,0 & $1,42(0,94-2,13)$ & & $1,42(0,88-2,26)$ & \\
\hline $4-5$ & 18,7 & $1,20(0,85-1,70)$ & & $1,11(0,74-1,66)$ & \\
\hline$\geq 6$ & 15,5 & 1,00 & & 1,00 & \\
\hline Cadastro no Programa Saúde da Família & & & 0,06 & & 0,05 \\
\hline Sim & 16,4 & 1,00 & & 1,00 & \\
\hline Não & 27,5 & $1,68(0,98-2,85)$ & & $1,66(0,99-2,78)$ & \\
\hline Inscrição no Programa Bolsa Família & & & 0,05 & & 0,02 \\
\hline Sim & 14,9 & 1,00 & & 1,00 & \\
\hline Não & 20,1 & $1,35(1,00-1,81)$ & & $1,57(1,07-2,30)$ & \\
\hline
\end{tabular}

* Salário mínimo na época: $\mathrm{R} \$ 350,00$;

** Teste de tendência.

associados. Das oito variáveis que compunham o bloco I, apenas a renda per capita familiar ( $\mathrm{p}=0,01)$ e número de moradores por cômodos ( $\mathrm{p}=0,006)$ figuraram como variáveis explicativas após o ajuste final do modelo. Ao incorporar as variáveis relacionadas às mães (bloco II) verificase que a idade e a anemia da mãe se associaram à anemia dos filhos ( $\mathrm{p}<0,001)$. No bloco III, a variável idade das crianças manteve a significância $(p<0,001)$, evidenciando que o aumento da idade seria um fator de proteção em relação à ocorrência de anemia, com um risco cinco vezes maior para as crianças que se encontravam na faixa entre 6 e 11 meses quando comparadas aquelas na faixa de 48 a 59 meses de vida.

A relação inversa entre anemia e idade das crianças persistiu no modelo final ajustado, sugerindo que o aumento da idade é um fator de proteção para a ocorrência de anemia.

\section{Discussão}

Em termos de magnitude, as prevalências de anemia em mães do Estado de Pernambuco $(16,4 \%)$ situam o problema como leve, segundo critérios da OMS, enquanto a ocorrência de 34,4\% em seus filhos menores de cinco anos enquadraria esta situação como um problema moderado de saúde pública 2 . Verifica-se, portanto, que mães e filhos apresentariam condições distintas, representando, assim, uma primeira diferenciação de situações em escala epidemiológica.

Por outra parte, os resultados se aproximam, da relação entre crianças e mulheres encontrada na África, nas Américas, no Sudeste da Ásia e no Mediterrâneo Oriental, num estudo de consolidação de dados pela OMS 1 em seis regiões do mundo, envolvendo 192 países, no período de 1993 a 2005; enquanto no Pacífico Ocidental e na Europa as frequências de anemia em préescolares e mulheres em idade reprodutiva, não grávidas, praticamente se equivalem. Essas distribuições poderiam configurar dois cenários epidemiológicos em nível mundial, um mais característico das populações radicadas em espaços geográficos de condições socioeconômicas mais desfavoráveis (no qual se enquadraria a situação de Pernambuco e do Brasil na década de 90), onde predominaria a prevalência de anemia nos menores de cinco anos, e outro cenário, representativo dos países mais desenvolvidos da 
Tabela 4

Distribuição da anemia e da razão de prevalência (RP) bruta em crianças menores de cinco anos segundo características biológicas, sócio-demográficas e ambientais. Pernambuco, Brasil, 2006.

\begin{tabular}{|c|c|c|c|c|}
\hline Blocos/Variáveis & $\begin{array}{c}\text { Amostra }(n=1.242) \text { * } \\
n\end{array}$ & $\begin{array}{c}\text { Anemia } \\
\%\end{array}$ & $\mathrm{RP}_{\text {bruta }}(\mathrm{IC} 95 \%)$ & Valor de p \\
\hline \multicolumn{5}{|l|}{ Bloco I } \\
\hline \multicolumn{5}{|l|}{ Fatores socioeconômicos } \\
\hline Renda per capita familiar (salários mínimos) ** & & & & 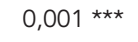 \\
\hline$<0,25$ & 649 & 38,2 & $1,32(0,83-2,11)$ & \\
\hline $0,25 \vdash 0,5$ & 373 & 33,0 & $1,14(0,71-1,85)$ & \\
\hline $0,5 \vdash 1,0$ & 151 & 21,9 & $0,76(0,44-1,31)$ & \\
\hline$\geq 1$ & 45 & 28,9 & 1,00 & \\
\hline Escolaridade materna (anos) & & & & $0,03 * \star \star$ \\
\hline 0 & 89 & 37,1 & $1,39(1,00-1,93)$ & \\
\hline 1 -5 & 454 & 35,2 & $1,32(1,05-1,66)$ & \\
\hline 5 卜9 & 393 & 38,9 & $1,46(1,17-1,83)$ & \\
\hline$\geq 9$ & 300 & 26,7 & 1,00 & \\
\hline \multicolumn{5}{|l|}{ Fatores ligados ao domicílio } \\
\hline Espaço geográfico & & & & 0,15 \\
\hline Urbano & 623 & 32,4 & 1,00 & \\
\hline Rural & 619 & 36,3 & $1,12(0,96-1,31)$ & \\
\hline Número de moradores por cômodo & & & & $<0,001$ *** \\
\hline$<1$ & 502 & 30,3 & 1,00 & \\
\hline $1 \mid-2$ & 585 & 35,0 & $1,16(0,97-1,38)$ & \\
\hline $2 \vdash 3$ & 108 & 41,7 & $1,38(1,06-1,78)$ & \\
\hline$\geq 3$ & 47 & 53,2 & $1,76(1,30-2,37)$ & \\
\hline Tipo de parede & & & & 0,02 \\
\hline Alvenaria/Tijolo & 1.155 & 33,6 & 1,00 & \\
\hline Outro & 87 & 44,8 & $1,33(1,04-1,71)$ & \\
\hline Tipo de piso & & & & 0,005 \\
\hline Cerâmica/Lajota & 238 & 25,6 & 1,00 & \\
\hline Cimento & 934 & 36,5 & $1,42(1,13-1,80)$ & \\
\hline Outro & 70 & 35,7 & $1,39(0,95-2,04)$ & \\
\hline Abastecimento de água & & & & 0,15 \\
\hline Rede geral & 683 & 32,7 & 1,00 & \\
\hline Outro & 559 & 36,5 & $1,12(0,96-1,30)$ & \\
\hline \multicolumn{5}{|l|}{ Esgotamento sanitário } \\
\hline Rede geral & 405 & 29,6 & 1,00 & 0,01 \\
\hline Fossa com tampa & 405 & 34,8 & $1,18(0,96-1,44)$ & \\
\hline Outro & 430 & 38,1 & $1,29(1,06-1,56)$ & \\
\hline \multicolumn{5}{|l|}{ Bloco II } \\
\hline \multicolumn{5}{|l|}{ Fatores maternos } \\
\hline Idade da mãe (anos) & & & & $<0,001$ *** \\
\hline$<20$ & 119 & 53,8 & $1,82(1,46-2,26)$ & \\
\hline $20-29,9$ & 661 & 34,2 & $1,15(0,97-1,38)$ & \\
\hline$\geq 30$ & 456 & 29,6 & 1,00 & \\
\hline Anemia da mãe $(\mathrm{Hb})$ & & & & $<0,001$ \\
\hline Anêmicas (<12g/dL) & 193 & 46,1 & $1,44(1,21-1,72)$ & \\
\hline Não anêmicas ( $\geq 12 \mathrm{~g} / \mathrm{dL}$ ) & 1.002 & 31,9 & 1,00 & \\
\hline Número de consultas no pré-natal & & & & 0,02 *** \\
\hline$\leq 3$ & 140 & 42,9 & $1,31(1,06-1,63)$ & \\
\hline $4-5$ & 241 & 35,7 & $1,09(0,90-1,33)$ & \\
\hline$\geq 6$ & 814 & 32,7 & 1,00 & \\
\hline
\end{tabular}

(continua) 
Tabela 4 (continuação)

\begin{tabular}{|c|c|c|c|c|}
\hline Blocos/Variáveis & $\begin{array}{c}\text { Amostra }(n=1.242) \text { * } \\
n\end{array}$ & $\begin{array}{c}\text { Anemia } \\
\%\end{array}$ & $\mathrm{RP}_{\text {bruta }}$ (IC95\%) & Valor de $p$ \\
\hline \multicolumn{5}{|l|}{ Bloco II } \\
\hline \multicolumn{5}{|l|}{ Serviços de saúde/Programas sociais } \\
\hline Cadastro no Programa Saúde da Família & & & & 0,37 \\
\hline Sim & 854 & 35,2 & 1,00 & \\
\hline Não & 51 & 41,2 & $1,17(0,83-1,64)$ & \\
\hline Inscrição no Programa Bolsa Família & & & & 0,92 \\
\hline Sim & 900 & 34,2 & 1,00 & \\
\hline Não & 336 & 34,5 & $1,01(0,85-1,20)$ & \\
\hline \multicolumn{5}{|l|}{ Bloco III } \\
\hline \multicolumn{5}{|l|}{ Fatores ligados à criança } \\
\hline Sexo & & & & 0,93 \\
\hline Masculino & 632 & 34,5 & 1,00 & \\
\hline Feminino & 610 & 34,3 & $0,99(0,85-1,16)$ & \\
\hline Idade (meses) & & & & $<0,001$ *** \\
\hline $6-11$ & 134 & 63,4 & $5,26(3,67-7,54)$ & \\
\hline $12-23$ & 305 & 56,1 & $4,65(3,28-6,60)$ & \\
\hline $24-35$ & 263 & 34,2 & $2,84(1,95-4,13)$ & \\
\hline $36-47$ & 291 & 17,5 & $1,45(0,96-2.21)$ & \\
\hline $48-59$ & 249 & 12,0 & 1,00 & \\
\hline Peso ao nascer (g) & & & & $0,81 * \star \star$ \\
\hline$<2.500$ & 113 & 37,2 & $1,07(0,83-1,39)$ & \\
\hline 2.500 -3.000 & 220 & 33,2 & $0,96(0,78-1,18)$ & \\
\hline$\geq 3.000$ & 880 & 34,7 & 1,00 & \\
\hline
\end{tabular}

$\mathrm{Hb}$ : hemoglobina.

* Os totais parciais de cada grupo podem diferir do total da amostra em função de informações ignoradas pelas entrevistadas. No caso específico de cadastro no Programa Saúde da Família, 27,2\% da amostra residiam em áreas não atendidas pelo programa;

** Salário mínimo na época: $\mathrm{R} \$ 350,00$;

*** Teste de tendência.

Europa e do Pacífico Ocidental, nos quais os riscos de anemia nos grupos biológicos de maior vulnerabilidade (pré-escolares e mulheres no período reprodutivo) passariam a se equivaler.

É evidente que essa visão mundial representa uma perspectiva de análise um tanto simplificada, na medida em que estabelece modelos para blocos geoeconômicos, continentais ou regionais (as chamadas regiões de saúde da OMS), compostos por vários países não necessariamente homogêneos, como no caso das Américas. De fato, as Américas representariam dois conjuntos bem distintos: os países de língua inglesa (Canadá e Estados Unidos) sendo que o último já consegue manter a anemia das crianças controlada 21 e, de outra parte, a América Latina, bastante heterogênea, onde o Chile aparece como o único a não apresentar anemia em mulheres como um problema de saúde pública ${ }^{1}$.

No caso do Brasil, estudos publicados entre 1992 e 2007 não eram conclusivos para consolidar um quadro informativo da situação da anemia em mulheres no período reprodutivo e em crianças menores de cinco anos ${ }^{5}$. Até então, estudos sobre crianças em algumas localidades, eram indicativos de prevalências acima de $40 \%$ 22,23,24,25. Nesses percentuais, os resultados expressariam a anemia como um grave problema de saúde pública 2. No entanto, o inquérito nacional Pesquisa Nacional de Demografia e Saúde da Criança e da Mulher realizado em 2006 (PNDS/2006) 26, numa amostra de 3.455 menores de cinco anos, encontrou uma prevalência de 20,9\% de anemia, com as maiores ocorrências no Nordeste $(25,5 \%)$ e uma distribuição praticamente equivalente no meio rural e urbano.

Já para as mulheres no período reprodutivo, no mesmo espaço de tempo, a prevalência de anemia foi estimada em torno de $20 \%$, metade do valor médio calculado para as crianças 22,27 . O estudo mais recente, PNDS/2006 26, mostra que as mulheres no período reprodutivo apresentaram uma prevalência de anemia de $29,4 \%$, quase $50 \%$ acima do encontrado para as crianças, e bem aci- 
Tabela 5

Distribuição percentual da anemia em menores de cinco anos, segundo as variáveis * que permaneceram no modelo final, com razão de prevalência (RP) bruta e ajustada. Pernambuco, Brasil, 2006.

\begin{tabular}{|c|c|c|c|c|c|}
\hline Blocos/Variáveis & $\begin{array}{c}\text { Anemia } \\
\%\end{array}$ & $\mathrm{RP}_{\text {bruta }}(\mathrm{IC} 95 \%)$ & Valor de p & RP ajustada (IC95\%) & Valor de p \\
\hline \multicolumn{6}{|l|}{ Bloco I } \\
\hline Renda per capita familiar (salários mínimos) ** & & & $0,001 * \star \star$ & & $0,01 * \star \star$ \\
\hline$<0,25$ & 38,2 & $1,32(0,83-2,11)$ & & $1,23(0,77-1,98)$ & \\
\hline $0,25 \vdash 0,5$ & 33,0 & $1,14(0,71-1,85)$ & & $1,11(0,69-1,79)$ & \\
\hline $0,5 \vdash 1,0$ & 21,9 & $0,76(0,44-1,31)$ & & $0,76(0,44-1,31)$ & \\
\hline$\geq 1$ & 28,9 & 1,00 & & 1,00 & \\
\hline Número de moradores por cômodo & & & $<0,001 * \star \star$ & & $0,006 * \star \star$ \\
\hline$<1$ & 30,3 & 1,00 & & 1,00 & \\
\hline 1 -2 & 35,0 & $1,16(0,97-1,38)$ & & $1,07(0,80-1,28)$ & \\
\hline-3 & 41,7 & $1,38(1,06-1,78)$ & & $1,25(0,95-1,63)$ & \\
\hline$\geq 3$ & 53,2 & $1,76(1,30-2,37)$ & & $1,59(1,17-2,17)$ & \\
\hline \multicolumn{6}{|l|}{ Bloco II } \\
\hline Idade da mãe (anos) & & & $<0,001 * \star \star$ & & $<0,001 * \star \star *$ \\
\hline$<20$ & 53,8 & $1,82(1,46-2,26)$ & & $1,89(1,52-2,36)$ & \\
\hline $20-29,9$ & 34,2 & $1,15(0,97-1,38)$ & & $1,20(1,00-1,43)$ & \\
\hline$\geq 30$ & 29,6 & 1,00 & & 1,00 & \\
\hline Anemia da mãe (Hb) & & & $<0,001$ & & $<0,001$ \\
\hline$<12 \mathrm{~g} / \mathrm{dL}$ & 46,1 & $1,44(1,21-1,72)$ & & $1,39(1,16-1,66)$ & \\
\hline$\geq 12 \mathrm{~g} / \mathrm{dL}$ & 31,9 & 1,00 & & 1,00 & \\
\hline \multicolumn{6}{|l|}{ Bloco III } \\
\hline Idade da criança (meses) & & & $<0,001 * \star \star$ & & $<0,001 * \star \star$ \\
\hline 6 -12 & 63,4 & $5,26(3,67-7,54)$ & & $5,01(3,47-7,24)$ & \\
\hline $12 \mid-24$ & 56,1 & $4,65(3,28-6,60)$ & & $4,47(3,12-6,40)$ & \\
\hline $24+36$ & 34,2 & $2,84(1,95-4,13)$ & & $2,78(1,89-4,07)$ & \\
\hline $36+48$ & 17,5 & $1,45(0,96-2.21)$ & & $1,36(0,89-2,09)$ & \\
\hline $48-59$ & 12,0 & 1,00 & & 1,00 & \\
\hline
\end{tabular}

$\mathrm{Hb}$ : hemoglobina.

* Variáveis que perderam a significância quando do ajuste do modelo: anos de estudo da mãe, espaço geográfico, tipo de parede, tipo de piso, abastecimento de água, esgotamento sanitário e número de consultas no pré-natal;

** Salário mínimo na época: $\mathrm{R} \$ 350,00$;

*** Teste de tendência.

ma dos valores que poderiam representar o comportamento médio de estudos anteriores. Seria, portanto, uma mudança surpreendentemente ilustrativa de novas tendências epidemiológicas.

$\mathrm{O}$ inquérito realizado em Pernambuco em 2006 representa, nesse aspecto, uma oportunidade ímpar para se estabelecer uma análise comparativa com o quadro anteriormente estudado nesse estado em 1997 12. Teria ocorrido, assim, uma evolução positiva do quadro epidemiológico, desde que a prevalência de anemia, baixou de $46,9 \%$ para $34,4 \%$ entre crianças e de $21,8 \%$ para $16,4 \%$ entre as mães. Essa evolução, entre outros fatores, poderia ser atribuída à implementação do programa de enriquecimento das massas ali- mentares de elevado consumo popular (trigo e farinha de milho) com ferro e folato, a partir de 2004 8, além da intensificação dos cuidados básicos de saúde de crianças e de gestantes, com o programa de suplementação de ferro medicamentoso pelos serviços de saúde.

Por outro lado, os resultados de Pernambuco, apresentam marcantes diferenças de tendências quando comparados com os dados da PNDS/2006 em escala nacional, em que, a prevalência de anemia entre as mulheres nordestinas $(39,1 \%)$ contrasta com a situação de mulheres avaliadas no mesmo ano em Pernambuco $(16,4 \%)$. Para as crianças as frequências de anemia foram, respectivamente, $25,5 \%$ e $34,4 \%$, para 
o Nordeste e Pernambuco, não se dispondo de elementos que expliquem estas diferenças.

Apesar de não se constituir um dos objetivos deste estudo, o caráter preliminar e especulativo da possível associação entre a implementação do programa de enriquecimento das farinhas de trigo e milho, diante da nova situação da anemia em mães e filhos no Estado de Pernambuco, justifica a sua realização. Portanto, parece pertinente e oportuno referir que outros estudos, especificamente realizados para responder a questão colocada anteriormente, tenham chegado a resultados conflitivos, existindo relato de um efeito positivo desta estratégia 28 , enquanto outro não detectou este impacto 29 .

No que se refere à associação entre a ocorrência de anemia em mães e filhos biológicos apenas um estudo no Brasil, em Pernambuco 12 e dois outros efetuados na Ásia Central 30 e no Egito 31 analisaram essa relação. No estudo de Pernambuco, em 1997, observou-se que mães com anemia têm uma chance estatisticamente mais elevada de terem filhos menores de cinco anos, na mesma condição. Detectou-se, ademais, que a partir dos três anos de idade, as prevalências de anemia em mães e filhos tornavam-se equivalentes, diferindo significantemente no grupo de menores de 36 meses 12. Esse comportamento se repetiu no presente estudo, mesmo com as reduções de prevalências registradas entre 1997 e 2006. Com respeito às duas investigações realizadas fora do Brasil, na Ásia Central (Cazaquistão, Uzbequistão e República Quirguiz), Sharmanov 30 observou que, das mães que apresentaram anemia severa, $68 \%$ dos filhos eram também portadores de anemia severa ou moderada, enquanto para o grupo de mães não anêmicas, o problema foi encontrado em apenas $24 \%$ dos filhos. Trata-se de uma pesquisa peculiarmente importante, por estudar clusters de três países com metodologias padronizadas, compreendendo 11.750 mães e seus respectivos filhos, com idade abaixo de três anos. Em estudo realizado no Alto Egito, El-Sayed et al. 31 encontraram um risco duas vezes maior de a criança ter anemia quando sua mãe era anêmica, razão que praticamente foi a mesma encontrada no estudo realizado em Pernambuco 12. É evidente que esses resultados não permitem uma extrapolação mais genérica para tipificar uma tendência em nível mundial.

Embora considerada como carência nutricional específica, com a deficiência de ferro representando o nutriente hematopoiético mais importante em sua etiologia 2, a anemia carencial, como outras deficiências nutricionais, representa um problema de determinação complexa 3,4 . Nessas circunstâncias, torna-se interessante recorrer a modelos explicativos multivariados co- mo forma de compreender a relação, interação e possível ordenação hierárquica dos fatores articulados na sua ocorrência, bem como esclarecer efeitos de confundimento que podem resultar nas análises univariadas 19,20.

Para analisar os possíveis papéis dos diversos fatores que podem influir na determinação dos resultados, os autores, partindo de variadas concepções, dos contextos da população pesquisada, utilizam diferentes modelos explicativos do problema. Esta diversidade de enfoques representa uma dificuldade preliminar para a comparação dos resultados entre os estudos efetuados.

No caso de Pernambuco, o modelo hierarquizado não foi aplicado para a análise de anemia nas mães, desde que apenas cinco variáveis independentes foram significativas no nível estatístico adotado $(p<0,20)$, para a seleção dos fatores a serem incluídos na análise multivariada. Dessas variáveis, após os ajustes, três permaneceram no modelo final: renda per capita familiar, cadastro no PSF e inscrição no PBF. Portanto, pela primeira vez, no caso de Pernambuco, O PBF e PSF passam a figurar na análise dos determinantes da anemia, observando-se que o primeiro (PBF) pode participar do modelo explicativo por conta do seu papel na formação da renda e, sobretudo, por sua importância como promotor das ações de saúde. Também em Pernambuco, Silva et al. 12 encontraram quatro fatores significativamente associados à anemia das mães (assistência prénatal, distância entre residência e serviço de saúde, destino do lixo e regime de ocupação do domicílio). No entanto, no período do referido estudo, não existia o PBF, enquanto o PSF ainda não atingia uma cobertura efetivamente significativa. No Rio Grande do Sul, Fabian et al. 27 observaram na análise multivariada que apenas a cor da pele, permaneceu no modelo final, verificando que as mulheres negras apresentaram um risco 3,17 maior de anemia em comparação com as brancas.

Mesmo fora do Brasil, são escassos os estudos aplicando modelos de análises multivariadas para compreender os fatores de determinação de anemia em mulheres e crianças. Shamah-Levy et al. 32, estudando 17.194 mulheres mexicanas, encontraram que no conjunto de variáveis significativamente associadas à anemia (área geográfica, idade, paridade, escolaridade, estrato socioeconômico, etnia), apenas a região geográfica (urbana/rural), paridade e condição socioeconômica foram mantidas no modelo final.

Para as crianças, dos fatores associados à anemia com $\mathrm{p}<0,20$ que entraram na análise multivariada hierarquizada, somente renda per capita familiar, número de moradores por cômodos, idade da mãe, anemia da mãe e idade 
da criança foram mantidos no modelo. Em relação ao estudo de Silva et al. 12, dentre os fatores considerados nos dois estudos, com exceção do espaço geográfico, esgotamento sanitário e número de consultas no pré-natal, todos os outros foram também significativos nas análises univariadas, podendo significar que continuariam a fazer parte de fatores potenciais de risco para anemia nas crianças.

Em Pelotas, Rio Grande do Sul, Assunção et al. 20 , utilizando níveis hierárquicos de determinação da anemia em amostra de 534 crianças menores de cinco anos, demonstraram que, após ajustadas, apenas a idade da criança e a renda familiar permaneceram estatisticamente significativas.

Em Alagoas, estudo de Vieira 25, envolvendo 768 menores de cinco anos e utilizando um conjunto de variáveis bem semelhantes ao aplicado em Pernambuco, encontrou na análise multivariada associação entre a anemia e a idade da criança, sendo a prevalência bem mais elevada nos menores de 36 meses, tal como no estudo aqui apresentado. Por outro lado, as variáveis, falta de assistência pré-natal e baixo peso ao nascer, que no estudo de Vieira 25 se relacionaram à ocorrência de anemia, em Pernambuco não foi encontrada associação destas variáveis com a anemia das crianças.

De modo análogo às observações do presente estudo, a dicotomia urbano/rural, que era historicamente bem diferente com franca predominância nas crianças do campo, passa a se equivaler no Nordeste depois de 2006 12,24,25,26. É admissível, portanto, considerar que tenha ocorrido uma marcante aproximação de resultados entre os dois espaços geográficos. Claro que há necessidade de novos estudos para confirmar essa possibilidade, que poderia configurar uma das manifestações recentes da rápida transição nutricional.

No que diz respeito à validade externa, chama a atenção a diversidade dos resultados relacionados a tempo, espaço geográfico e pessoas na comparação com os resultados deste estudo, sendo que as diferenças são compatíveis com as marcantes mudanças de situações.

Numa visão sintética, em razão da escassez de pesquisas, diversidade de procedimentos amostrais e métodos de avaliação da anemia em mães e filhos, não se tem ainda uma acumulação consistente de resultados e conclusões sobre o problema. A impressão dominante é que, mesmo com a discrepância de ocorrências entre mães e filhos, prevalece a observação de que os dois eventos acham-se associados, em maior ou menor grau. Já o inventário analítico dos fatores de risco parece bem mais inconsistente. Assim, em relação à anemia, apenas a renda representou um fator comum de risco para mães e crianças em Pernambuco, no estudo aqui analisado, representando, portanto, evidentes diferenças em relação aos resultados descritos por Silva et al. 12, analisando a situação existente em 1997. Por outro lado, outras investigações efetuadas no Nordeste e no Brasil, sem o pareamento mães/filhos biológicos, observado neste estudo, embora coincidindo pontualmente em alguns aspectos (renda, condições de moradia, escolaridade das mães) apresentam mais evidências de desencontros que de concordâncias, na identificação de fatores de riscos. Essa parece ser a avaliação que prevalece na comparação dos resultados, deixando em aberto um grande elenco de alternativas a serem investigadas. Mães e filhos, além da parceria biológica da vida intra-uterina, têm, no período de amamentação, um prolongamento do processo nutricional, que se traduz na dependência mamária.

Esses mecanismos biológicos estabelecem um primeiro "compromisso" de interação mãe-filho que no caso específico das anemias, é interrompido ou desequilibrado pelos hábitos alimentares, estilos de vida, constrangimentos da realidade socioeconômica e adversidades ambientais. Estabelece-se, assim, um contexto de risco que assume diferentes configurações em cada situação e que, de fato, só agora começa a ser devidamente estudado de uma forma ainda incompleta, preliminar e pouco sistematizada. É o caso, por exemplo, das condições alimentares do binômio mãe-filho, durante a pré-concepção, a gravidez, a lactação e o pós-desmame, estabelecendo uma cadeia de eventos que não tem sido devidamente considerada, seja no plano de sua validade interna, seja, sobretudo, na instância mais ampla de sua extrapolação. 


\section{Resumo}

Analisaram-se prevalência e fatores associados à anemia em mães $(n=1.022)$ e seus filhos $(n=1.242)$ menores de 5 anos em Pernambuco, Brasil, 2006. Estudo transversal, populacional, com amostra probabilística representativa do meio urbano e rural. Diagnóstico de anemia determinado pelo nível de hemoglobina $<11 \mathrm{~g} /$ $d L$ (crianças) $e<12 \mathrm{~g} / d L$ (mulheres), dosagem feita pelo HemoCue. Análises uni e multivariadas realizadas por regressão de Poisson com ajuste robusto do erro padrão, adotando-se para crianças modelo hierárquico de determinação do desfecho. Este procedimento não foi aplicado às mães, pelo pequeno número de fatores associados nas análises univariadas. Prevalência de anemia foi 16,4\% e 34,4\%, em mães e filhos, respectivamente. Mães anêmicas comparadas às mães com níveis normais de hemoglobina apresentaram $R P=1,44$ (IC95\%: 1,21-1,72) em relação ao risco de anemia em seus filhos, praticamente se mantendo no modelo ajustado $(R P=1,39$; IC95\%: 1,16-1,66). A prevalência de anemia nos filhos foi o dobro da registrada nas mães, encontrando-se, no modelo final, apenas um único fator comum para ambos: renda per capita familiar.

Anemia; Mães; Prevalência

\section{Colaboradores}

T. C. Miglioli participou da pesquisa bibliográfica, processamento e análise de dados e redação do texto. A. M Brito colaborou na concepção e coordenação da análise dos dados, redação e revisão final do texto. P. I. C. Lira contribuiu na coordenação do projeto, redação e revisão final do texto. J. N. Figueroa participou da análise e interpretação dos dados. M. Batista Filho participou da concepção e coordenação do projeto, redação e revisão final do texto.

\section{Agradecimentos}

Ao Conselho Nacional de Desenvolvimento Científico e Tecnológico (CNPq) pelo financiamento.

\section{Referências}

1. World Health Organization. Worldwide prevalence of anaemia 1993-2005: WHO global database on anaemia. Geneva: World Health Organization; 2008.

2. World Health Organization. Iron deficiency anaemia: assessment, prevention and control: a guide for programme managers. Geneva: World Health Organization; 2001.

3. Stoltzfus RJ. Iron deficiency: global prevalence and consequences. Food Nutr Bull 2003; 24(4 Suppl):S99-103

4. World Health Organization. Focusing on anaemia: towards an integrated approach for effective anaemia control. Geneva: World Health Organization; 2004.
5. Lira PIC, Ferreira LOC. Epidemiologia da anemia ferropriva. In: Kac G, Schieri R, Gigante DP, organizadores. Epidemiologia nutricional. Rio de Janeiro: Editora Fiocruz/São Paulo: Editora Atheneu; 2007. p. 445-60.

6. Fundo das Nações Unidas para a Infância. Estratégia para melhorar a nutrição de crianças e mulheres nos países em desenvolvimento. Nova York: Fundo das Nações Unidas para a Infância; 1990.

7. Ministério da Saúde. Compromisso social para a redução da anemia por carência de ferro no Brasil. http://nutricao.saude.gov.br/mn/ferro/docs/ compromisso_social_reducao_anemia.pdf (acessado em Out/2008). 
8. Agência Nacional de Vigilância Sanitária. Resolução RDC nº. 344, de 13 de dezembro de 2002. Regulamento técnico para fortificação das farinhas de trigo e das farinhas de milho com ferro e ácido fólico. Diário Oficial da União 2002; 18 dez.

9. Brasil. Portaria n ${ }^{\circ} .730$, de 13 de maio de 2005. Diário Oficial da União 2005; 16 mai.

10. Engstrom EM, Anjos LA. Relação entre o estado nutricional materno e sobrepeso nas crianças brasileiras. Rev Saúde Pública 1996; 30:233-9.

11. Engstrom EM, Anjos LA. Déficit estatural nas crianças brasileiras: relação com condições sócioambientais e estado nutricional materno. Cad Saúde Pública 1999; 15:559-67.

12. Silva SCL, Batista Filho M, Miglioli TC. Prevalência e fatores de risco de anemia em mães e filhos no Estado de Pernambuco. Rev Bras Epidemiol 2008; 11:266-77.

13. Ministério da Saúde. Saúde da família: resultados alcançados em 2007 (físico e financeiro). http://200.214.130.35/dab/abnumeros.php (acessado em Ago/2010).

14. Ministério do Desenvolvimento Social e Combate à Fome. Programa Bolsa Família: principais resultados. http://www.mds.gov.br/bolsafamilia/o_ programa_bolsa_familia/principais-resultados (acessado em Mar/2009).

15. Monteiro CA, Mondini L, Souza ALM, Popkin BM. Da desnutrição para a obesidade: a transição nutricional no Brasil. In: Monteiro CA, organizador. Velhos e novos males da saúde no Brasil. São Paulo: Editora Hucitec; 2000. p. 247-55.

16. Batista Filho M, Assis AMO, Kac G. Transição nutricional: conceitos e características. In: Kac G, Sichieri R, Gigante DP, organizadores. Epidemiologia nutricional. Rio de Janeiro: Editora Fiocruz/São Paulo: Editora Atheneu; 2007. p. 445-60.

17. Batista Filho M, Romani SAM, organizadores. Alimentação, nutrição e saúde no Estado de Pernambuco: espacialização e fatores sócio-econômicos. Recife: Instituto de Medicina Integral Prof. Fernando Figueira; 2002. (Série de Publicações Científicas do IMIP, 7).

18. Barros AJ, Hirakata VN. Alternatives for logistic regression in cross-sectional studies: an empirical comparison of models that directly estimate the prevalence ratio. BMC Med Res Methodol 2003; 3:21.

19. Silva LSM, Giugliani ERJ, Aerts DRGC. Prevalência e determinantes de anemia em crianças de Porto Alegre, RS, Brasil. Rev Saúde Pública 2001; 35:66-73.

20. Assunção MCF, Santos IS. Barros AJD, Gigante DP Victora CG. Anemia em menores de seis anos: estudo de base populacional em Pelotas, RS. Rev Saúde Pública 2007; 41:328-35.
21. Centers for Diseases Control and Prevention. Iron deficiency - United Estates, 1999-2000. MMWR Morb Mortal Wkly Rep 2002; 51:897-9.

22. Governo do Estado do Piauí/Universidade Federal do Piauí/Universidade Federal de Pelotas/Fundo das Nações Unidas para a Infância. Adolescentes no Piauí: saúde, educação e trabalho. Brasília: Fundo das Nações Unidas para a Infância; 1992.

23. Monteiro CA, Szarfarc SC, Mondini L. Tendência secular da anemia na infância na cidade de São Paulo (1984-1996). Rev Saúde Pública 2000; 34 Suppl 6:S62-72.

24. Osório MM, Lira PIC, Batista Filho M, Ashworth A. Prevalence of anemia in children 6-59 months old in the state of Pernambuco, Brazil. Rev Panam Salud Pública 2001; 10:101-7.

25. Vieira RCS. Fatores associados à prevalência de anemia em crianças menores de cinco anos do Estado de Alagoas, Brasil [Dissertação de Mestrado]. Maceió: Universidade Federal de Alagoas; 2007.

26. Ministério da Saúde. Pesquisa Nacional de Demografia e Saúde da Criança e da Mulher PNDS/2006. Brasília: Ministério da Saúde; 2008.

27. Fabian C, Olinto MTA, Dias-da-Costa JS, Bairros F Nácul LC. Prevalência de anemia e fatores associados em mulheres adultas residentes em São Leopoldo, Rio Grande do Sul, Brasil. Cad Saúde Pública 2007 ; 23:1199-205.

28. Costa CA, Machado EH, Colli C, Latorre WC, Szarfarc SC. Anemia em pré-escolares atendidos em creches de São Paulo (SP): perspectivas decorrentes da fortificação das farinhas de trigo e de milho. Nutrire Rev Soc Bras Aliment Nutr 2009; 34:59-74.

29. Assunção MCF, Santos IS, Barros AJD, Gigante DP, Victora CG. Efeito da fortificação de farinhas com ferro sobre anemia em pré-escolares, Pelotas, RS. Rev Saúde Pública 2007; 41:539-48.

30. Sharmanov A. Anaemia in Central Asia: demographic and health survey experience. Food Nutr Bull 1998; 19:307-17.

31. El-Sayed N, Gad A, Nofal L, Zeid HA, El-Morshedy $\mathrm{H}$, El-Waseef S. Assessment of the prevalence and potential determinants of nutritional anaemia in Upper Egypt. Food Nutr Bull 1999; 20:417-28.

32. Shamah-Levy T, Villalpando S, Rivera JA, MejíaRodríguez F, Camacho-Cisneros M, Monterrubio EA. Anemia in Mexican women: a public health problem. Salud Pública Méx 2003; 45 Suppl 4:S499507.

Recebido em 27/Jan/2010

Versão final reapresentada em 19/Jul/2010

Aprovado em 02/Ago/2010 